\title{
Improved Stability and Smart-Material Functionality Realized in an Energetic Cocrystal**
}

\author{
Onas Bolton and Adam J. Matzger*
}

Though energetic compounds, and explosives in particular, represent some of the most influential materials in human history, their modern evolution has been relatively slow. Aside from being hindered by the inherent dangers of the field, improving on the state-of-the-art is difficult as novel energetic materials (energetics) must achieve a challenging combination of properties including high explosive power, high stability, and low cost. Moreover, explosive power is highly dependent on solid-state density, and though chemical structures with high energies can be designed, engineering their crystal structures is not possible. In fact, crystal structure prediction, a problem closely related to crystal engineering, is fraught with challenges. ${ }^{[1]}$ This is especially so for compounds with nitro groups, ${ }^{[2]}$ a common moiety among energetic compounds.

Though an admirable amount of work has gone into the discovery of novel compounds with high chemical potential energy, ${ }^{[3]}$ few of these materials have proven viable for explosives applications. Work to improve the material properties of existing energetics has focused primarily on exploring polymorphism in hopes to find more dense or less sensitive forms. ${ }^{[4]}$ This approach is attractive because it allows one to improve energetic compounds without the daunting task of implementing new, safe, and scalable chemistry. This advantage is present in another approach that is only starting to garner interest in solid-state energetics research: cocrystallization. ${ }^{[5]}$

Until recently, cocrystallization had been absent from the literature as a method for energetic solid form engineering despite its current success in engineering solid forms of pharmaceuticals. ${ }^{[6]}$ This imbalance is perhaps due to the chemical differences distinguishing energetics and pharmaceuticals. Most active pharmaceutical ingredients feature polar groups that are rich in predictable interactions conducive to cocrystal formation: primarily hydrogen bonding. ${ }^{[7,8]}$ Energetics, in contrast, are defined primarily by nitro groups, a solitary moiety that offers very few predictable interactions sufficiently strong or versatile to be useful in cocrystal design..$^{[9]}$

[*] Dr. O. Bolton, Prof. A. J. Matzger

Department of Chemistry and the Macromolecular Engineering

Program, University of Michigan

Ann Arbor, MI 48109-1055 (USA)

E-mail:matzger@umich.edu

[**] This work was supported by the Defense Threat Reduction Agency (HDTRA1-09-1-0033). We thank the China Lake Naval Air Weapons Station for CL-20.

D) Supporting information for this article is available on the WWWW under http://dx.doi.org/10.1002/anie.201104164.
Recently, we demonstrated that cocrystallization can be used to generate novel solid forms of energetic materials by presenting seventeen cocrystals containing 2,4,6-trinitrotoluene (TNT) ${ }^{[10]}$ Though the work proved the viability of cocrystallization to increase density and improve thermal stability in energetic materials, these improvements were achieved to the detriment of explosive power. When cocrystallized with a non-energetic compound, as was the case with these TNT cocrystals, the energetic component inevitably sees its explosive power diluted. Several measureable material properties are improved, but the resultant cocrystals are not viable explosives. Moreover, all of these cocrystals were formed by $\pi-\pi$ stacking, a synthon available to the aromatic class of energetics, but not to the broader and more powerful non-aromatic class. Therefore, it remains unclear if energeticenergetic cocrystals can be realized based solely upon $\mathrm{C}-\mathrm{H}$ and nitro-group interactions.

Presented here is an energetic-energetic cocrystal composed of 2,4,6,8,10,12-hexanitro-2,4,6,8,10,12-hexaazaisowurtzitane (CL-20) and TNT in a 1:1 molar ratio (1, Figure 1). CL-20 is a relatively new energetic compound<smiles>Cc1c([N+](=O)[O-])cc([N+](=O)[O-])cc1[N+](=O)[O-]</smiles>

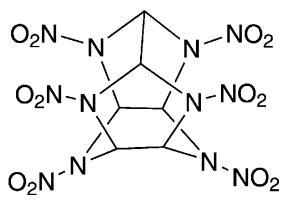

Figure 1.

developed by the United States Navy. ${ }^{[11]}$ A non-aromatic cyclic nitramine, it features high density, high detonation velocity (one measure of explosive power), and favorable oxygen balance. Disadvantages include high production costs and high sensitivity (it detonates readily when subjected to physical impact).${ }^{[12]}$ These issues have slowed the introduction of CL-20 into explosives applications, failing to meet cost and safety standards set by its cyclic nitramine cousins 1,3,5trinitroperhydro-1,3,5-triazine (RDX) and octahydro-1,3,5,7tetranitro-1,3,5,7-tetrazocine (HMX) ${ }^{[13]}$ two energetics that are long established and widely used explosives. Contrasting with CL-20 in many ways is TNT, which has poor density, low oxygen balance, and modest detonation velocity, but features low sensitivity to impact ${ }^{[14]}$ and economical production costs. TNT is also highly detectable and is, in fact, used very widely as a benchmark for energetic detection technologies. ${ }^{[15]}$

In cocrystal 1 the problem of effective dilution of the explosive is avoided. By being comprised solely of explosive components the resultant cocrystal retains the explosive 
properties of its constituents. Cocrystal $\mathbf{1}$ also exemplifies the capabilities of aliphatic, highly nitrated compounds to cocrystallize despite their lack of strong, predictable interactions. With no available $\pi-\pi$ stacking, this cocrystal forms based on a series of $\mathrm{CH}$ hydrogen bonds between nitro group oxygens and aliphatic hydrogens as well as interactions between the electron-deficient ring of TNT and nitro groups of CL-20. As such, this cocrystal sits at the interface between aromatic and aliphatic energetics, a common demarcation in the field, by combining members of each class into a single solid form.

Cocrystal 1 was formed by growth from saturated organic solutions as well as by solvent-mediated solid-state conversion. These cocrystals appear as thick colorless prisms easily distinguished from pure CL-20 and TNT polymorphs by their distinct habit (Figure 2). High-quality crystals were grown

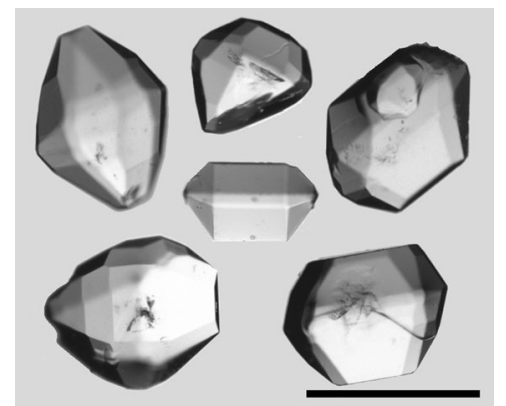

Figure 2. Prismatic habits of cocrystal 1 (scale bar is $500 \mu \mathrm{m}$ ).

from ethanol solution, and their crystal structure was determined by single-crystal X-ray diffraction. ${ }^{[16]}$ Cocrystal 1 is also easily distinguished from pure components by powder X-ray diffraction or Raman spectroscopy (Figure 3). Differ-
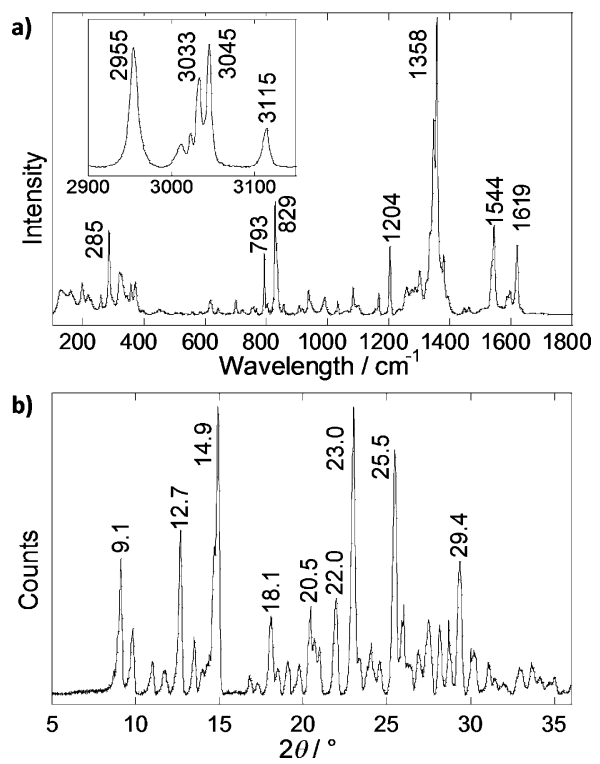

Figure 3. Identification of cocrystal 1. a) Raman spectrum measured with $647 \mathrm{~nm}$ laser with magnified high wavenumber region inset. b) Powder X-ray diffraction pattern, $\mathrm{Cu}_{\mathrm{K \alpha}}$ radiation. ential scanning calorimetry (DSC) of this cocrystal reveals that the cocrystal converts at $136^{\circ} \mathrm{C}$ to liquid TNT $\left(T_{\mathrm{m}}=81-\right.$ $82^{\circ} \mathrm{C}$ ) and $\beta$-CL-20 (confirmed by Raman spectroscopy.)

The crystal structure of $\mathbf{1}$ is defined exclusively by interactions involving nitro groups, the shortest being five $\mathrm{CH}$ hydrogen bonds that propagate through the crystal. The closest noncovalent interatomic contact lies between an imidazolidine CL-20 nitro group and the 3-position hydrogen of TNT, while the second shortest unites the 6-nitro group of that same TNT molecule to a hydrogen at the junction of the imidazolidine and piperazine rings of another CL-20 molecule. These interactions form a repeating zigzagging chain in the [010] direction (Figure 4a). The third and fourth shortest

a)
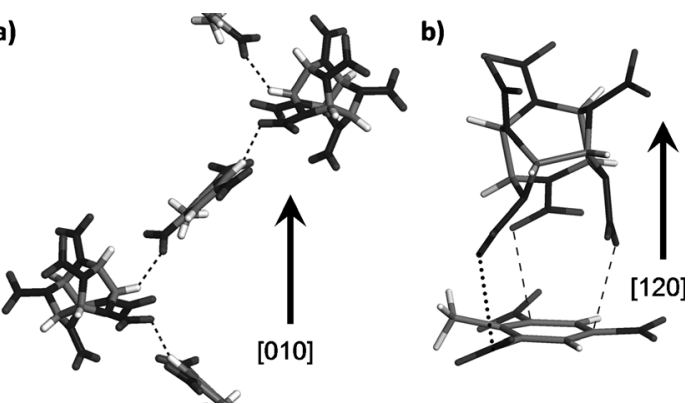

Figure 4. Interactions between CL-20 and TNT in 1. a) Propagating $\mathrm{CH}$ hydrogen bonding. b) Nonpropagating nitro-aromatic (dashed line) and nitro-nitro interactions (dotted).

contacts, also $\mathrm{CH}$ hydrogen bonds, appear between adjacent CL-20 molecules along with a fifth connecting the 5-position TNT hydrogen to another imidazolidine CL-20 nitro oxygen. These interactions stack the zigzag chains to complete the cocrystal. There are no significant TNT-TNT interactions in 1.

Cocrystal 1 exhibits two interactions between CL-20 nitro groups and the electron-poor ring of TNT (Figure 4b). These are somewhat analogous to the defining interactions of TNTaromatic cocrystals in which electron-rich rings of the cocrystal former stack with the electron-poor ring of TNT. $^{[10]}$ The interactions in $\mathbf{1}$ do not, however, appear to direct the crystal structure as they do not propagate through the crystal. The third CL-20 nitro group facing this TNT molecule donates an oxygen to the 2-nitro nitrogen atom of TNT in an $\mathrm{N}=\mathrm{O} \cdots \mathrm{NO}_{2}$ arrangement that appears frequently elsewhere in 1, though at lengths suggesting that these interactions are rather weak. These CL-20/TNT ring interactions are oriented approximately in the [120] direction. In addition to these directional interactions, molecular characteristics such as size, shape, and polarity are reported to assist in cocrystal formation and may contribute to stabilize $\mathbf{1}^{[17]}$

Cocrystal 1 has a crystallographic density of $1.91 \mathrm{~g} \mathrm{~cm}^{-3}$ at $95 \mathrm{~K}$, which is somewhat lower than those of CL-20 polymorphs $\left(1.95-2.08 \mathrm{~g} \mathrm{~cm}^{-3}\right),{ }^{[18]}$ but substantially higher than those of either monoclinic or orthorhombic TNT (1.70$\left.1.71 \mathrm{~g} \mathrm{~cm}^{-3}\right) \cdot{ }^{[19]}$ The packing coefficient of $\mathbf{1}$, measured as the ratio of total molecular volume to unit cell volume, is 0.802 , which is higher than CL-20 polymorphs $\alpha$ (anhydrous 0.640 ), 
$\beta$ (0.774), and $\gamma$ (0.762). This packing coefficient is lower, however, than those of pure TNT (monoclinic 0.824, orthorhombic 0.820 ) and $\varepsilon$-CL-20 (0.816). All forms of CL-20 and TNT were observed to convert to 1 during solvent-mediated experiments.

At $296 \mathrm{~K}$ the crystallographic density of $\mathbf{1}$ measures $1.84 \mathrm{~g} \mathrm{~cm}^{-3}$ as the unit cell exhibits thermal expansion in two of three dimensions. Relative to its unit cell at $95 \mathrm{~K}$, dimension $a$ expands from 9.67 to $9.77 \AA$ ( $+0.99 \%)$ and $b$ from 19.37 to $19.86 \AA$ ( $+2.53 \%)$, while $c$ remains unchanged, 24.69 to $24.70 \AA(+0.04 \%)$. Packing in the directions of cell dimensions $a$ and $b$ primarily involve the interactions depicted in Figure 4, those between CL-20 and TNT. Interactions in the [001] $(c)$ direction are predominantly those between adjacent CL-20 molecules (Figure 5).

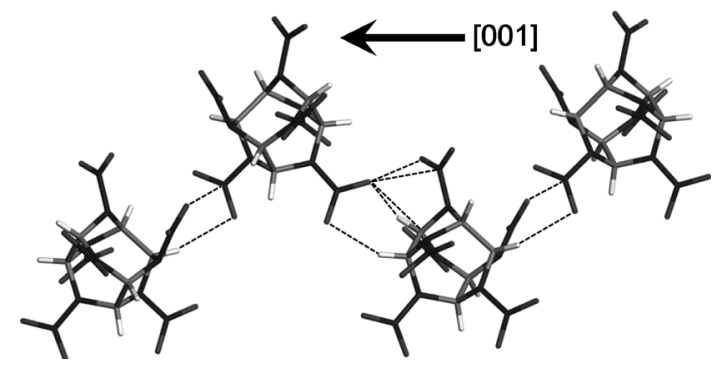

Figure 5. Nitro-hydrogen and nitro-nitro interactions between CL-20 molecules propagating throughout 1

Turning to the performance characteristics of $\mathbf{1}$, impact sensitivity was measured using a drop test. The device employed a $2940 \mathrm{~g}$ weight, stainless-steel impactor and anvil, and samples of one mg each sealed inside an aluminum DSC pan (to consolidate the sample and prevent sample ejection during testing). Thirty samples of each material were tested with $h_{50 \%}$ measured as the dropping height at which there was a $50 \%$ probability of detonation. An $h_{50 \%}$ of $47 \mathrm{~cm}$ was measured for $\varepsilon$-CL-20 while 1 exhibited an $h_{50 \%}$ of $99 \mathrm{~cm}$, more than twice the height of CL-20. ${ }^{[20]}$ Incorporating insensitive TNT into a cocrystal with sensitive CL-20 greatly reduces its impact sensitivity, potentially improving the viability of CL-20 in explosive applications.

The thermal properties of $\mathbf{1}$ make it a sensitivity-changeable energetic, a property that highlights the unique potential for cocrystallization to produce energetic smart materials. At $136^{\circ} \mathrm{C}$ cocrystal 1 converts to liquid TNT and either $\beta$ or $\gamma$ CL-20, depending on the degree of overheating. Cooling the resultant mixture does not lead to the recrystallizion of $\mathbf{1}$. Thus, an insensitive energetic that is not easily detonated by impact, $\mathbf{1}$, is transformed into a sensitive energetic that is readily detonated by impact, CL-20, simply through heating. Samples of 1, each of $1 \mathrm{mg}$ sealed in DSC pans just as in prior drop tests, were heated to $150{ }^{\circ} \mathrm{C}$ and held for $10 \mathrm{~min}$ to ensure complete conversion to their pure components. Samples were then cooled to ambient temperature. Drop tests on these "activated" samples yielded an $h_{50 \%}$ of $41 \mathrm{~cm}$ indicating greater sensitivity than $\varepsilon$-CL-20. This demonstrates the ability of cocrystallization to yield an energetic smart material that can be transported or stored in an insensitive form then converted to an activated form for deployment.

In conclusion, we have discovered and characterized an energetic-energetic cocrystal composed of a 1:1 molar ratio of established explosives CL-20 and TNT. This cocrystal forms readily despite the lack of strong predictable interactions in the chemical structures of its components and appears to be stabilized by a number of $\mathrm{CH}$ hydrogen bonds each involving nitro group oxygen atoms. This holds promise that more energetic-energetic cocrystals may be discovered from among the nitro-rich, non-aromatic compounds that dominate the field. Exemplifying the practical merits of energetic cocrystallization, this cocrystal combines the economy and stability of TNT with the density and power of CL-20 into a homogenous energetic with high explosive power and excellent insensitivity. An approximate doubling of the impact stability is achieved, relative to pure CL-20. Furthermore, the cocrystal demonstrates the potential of cocrystallization to realize explosive smart materials. Here, the impact-insensitive cocrystal becomes highly impact-sensitive simply by heat, making this cocrystal an energetic with "turn-on" sensitivity.

Received: June 16, 2011

Revised: July 12, 2011

Published online: August 25, 2011

Keywords: cocrystallization · crystal growth · explosives . Raman spectroscopy $\cdot$ solid-state structures

[1] a) C. W. Lehmann, Angew. Chem. 2011, 123, 5731-5732; Angew. Chem. Int. Ed. 2011, 50, 5616-5617; b) H. C. S. Chan, J. Kendrick, F. J. J. Leusen, Angew. Chem. 2011, 123, 3035-3037; Angew. Chem. Int. Ed. 2011, 50, 2979-2981; c) K. Merz, V. Vasylyeva, CrystEngComm 2010, 12, 3989-4002; d) S. Roy, A. J. Matzger, Angew. Chem. 2009, 121, 8657-8660; Angew. Chem. Int. Ed. 2009, 48, 8505-8508; e) G. M. Day et al., Acta Crystallogr. Sect. B 2009, 65, 107-125; f) G. R. Desiraju, Nat. Mater. 2002, 1, 77-79.

[2] a) S. A. Barnett, A. Johnston, A. J. Florence, S. L. Price, D. A. Tocher, Cryst. Growth Des. 2008, 8, 24-36; b) F. Demartin, G. Filippini, A. Gavezzotti, S. Rizzato, Acta Crystallogr. Sect. B 2004, 60, 609-620.

[3] a) J. Stierstorfer, K. R. Tarantik, T. M. Klapötke, Chem. Eur. J. 2009, 15, 5775-5792; b) R. Wang, H. Gao, C. Ye, B. Twamley, J. M. Shreeve, Inorg. Chem. 2007, 46, 932 -938; c) J. C. GálvezRuiz, G. Holl, K. Karaghiosoff, T. M. Klapötke, K. Löhnwitz, P. Mayer, H. Nöth, K. Polborn, C. J. Rohbogner, M. Suter, J. J. Weigand, Inorg. Chem. 2005, 44, 4237-4253; d) M. Zhang, P. E. Eaton, R. Gilardi, Angew. Chem. 2000, 112, 422-426; Angew. Chem. Int. Ed. 2000, 39, 401-404.

[4] a) Z. A. Dreger, Y. M. Gupta, J. Phys. Chem. A 2010, 114, 80998105 ; b) F. P. A. Fabbiani, C. R. Pulham, Chem. Soc. Rev. 2006, 35, $932-942$; c) J. Evers, T. M. Klapötke, P. Mayer, G. Oehlinger, J. Welch, Inorg. Chem. 2006, 45, 4996-5007; d) W. C. McCrone, Anal. Chem. 1950, 22, 1225-1226.

[5] Cocrystal defined: a) A. D. Bond, CrystEngComm 2007, 9, 833 834; examples: b) G. P. Stahly, Cryst. Growth Des. 2009, 9, 4212 4229; c) A. N. Sokolov, T. Friščić, L. R. MacGillivray, J. Am. Chem. Soc. 2006, 128, 2806-2807; d) C. B. Aakeröy, A. M. Beatty, B. A. Helfrich, Angew. Chem. 2001, 113, 3340-3342; Angew. Chem. Int. Ed. 2001, 40, 3240-3242; e) M. C. Etter, D. A. Adsmond, J. Chem. Soc. Chem. Commun. 1990, 589-591; 
f) M. C. Etter, P. W. Baures, J. Am. Chem. Soc. 1988, 110, 639640.

[6] a) T. Friščić, W. Jones, J. Pharm. Pharmacol. 2010, 62, $1547-$ 1559 ; b) C. B. Aakeröy, S. Forbes, J. Desper, J. Am. Chem. Soc. 2009, 131, $17048-17049$; c) N. Shan, M. J. Zaworotko, Drug. Discov. Today 2008, 13, 440-446; d) M. Wenger, J. Bernstein Angew. Chem. 2006, 118, 8134-8137; Angew. Chem. Int. Ed. 2006, 45, 7966-7969; e) J. F. Remenar, S. L. Morissette, M. L. Peterson, B. Moulton, J. M. MacPhee, H. R. Guzman, O. Almarsson, J. Am. Chem. Soc. 2003, 125, 8456-8457.

[7] G. R. Desiraju, Angew. Chem. 1995, 107, 2541-2558; Angew. Chem. Int. Ed. Engl. 1995, 34, 2311-2327.

[8] N. Blagden, D. J. Berry, A. Parkin, H. Javed, A. Ibrahim, P. T Gavan, L. L. De Matos, C. C. Seaton, New J. Chem. 2008, 32, $1659-1672$.

[9] a) C. V. Ramana, S. Chatterjee, K. A. Durugkar, R. G. Gonnade, CrystEngComm 2008, 11, 143-150; b) S. Adilov, V. R. Thalladi, Cryst. Growth Des. 2007, 7, 481-484; c) S. George, A. Nagaia, C.-K. Lam, T. C. W. Mak, J.-F. Nicoud, Chem. Commun. 2004, 1201-1203; d) P. K. Thallapally, S. Basavoju, G. R. Desiraju, M. Bagieu-Beucher, R. Masse, J.-F. Nicoud, Curr. Sci. India 2003, 85, $995-1001$; e) R. K. R. Jetti, P. K. Thallapally, A. Nangia, C.K. Lam, T. C. W. Mak, Chem. Commun. 2002, 952-953.

[10] K. B. Landenberger, A. J. Matzger, Cryst. Growth Des. 2010, 10, $5341-5347$.

[11] K. J. Kraeutle, JANNAF Proceedings. CPIA Publ. 1988 (October), 498.
[12] R. L. Simpson, P. A. Urtiew, D. L. Ornellas, G. L. Moody, K. J. Scribner, D. M. Hoffman, Propellants Explos. Pyrotech. 1997, 22, 249-255.

[13] B. M. Rice, J. J. Hare, J. Phys. Chem. A 2002, 106, 1770-1783.

[14] W. S. Wilson, D. E. Bliss, S. L. Christian, D. J. Knight, US Naval Weapons Center Technical Report 1990 NWC TP 7073.

[15] a) M. E. Germain, M. J. Knapp, Chem. Soc. Rev. 2009, 38, $2543-$ 2555; b) A. Rose, Z. Zhu, C. F. Madigan, T. M. Swager, V. Bulović, Nature 2005, 434, 876-879.

[16] Crystal data for cocrystal 1: $\mathrm{C}_{13} \mathrm{H}_{11} \mathrm{~N}_{15} \mathrm{O}_{18}, M_{r}=665.32$, crystal dimensions $0.29 \times 0.14 \times 0.16 \mathrm{~mm}^{3}$, orthorhombic, space group Pbca $, a=9.67390(18), b=19.3690(4), c=24.6898(17) \AA, \quad V=$ $4626.2(3) \AA^{3}, Z=8, \rho_{\text {calcd }}=1.910 \mathrm{~g} \mathrm{~cm}^{-3}, T=95 \mathrm{~K}, 19466$ measured, 4054 independent, 4054 observed $[(I)>2 \sigma(I)]$ reflections, $R_{\text {int }}=0.021, R_{1}=0.55, w R_{2}=0.242$ [for $(I)>2 \sigma(I)$ ], $S=1.045$. CCDC 826174 contains the supplementary crystallographic data for this paper. These data can be obtained free of charge from The Cambridge Crystallographic Data Centre via www.ccdc. cam.ac.uk/data_request/cif.

[17] L. Fábián, Cryst. Growth Des. 2009, 9, 1436-1443.

[18] Measured at $100 \mathrm{~K}$; N. B. Bolotina, M. J. Hardie, R. L. Speer, Jr., A. A. Pinkerton, J. Appl. Crystallogr. 2004, 37, 808-814.

[19] Measured at 123 K; R. M. Vrcelj, J. N. Sherwood, A. R. Kennedy, H. G. Gallagher, T. Gelbrich, Cryst. Growth Des. 2003, 3 , $1027-1032$.

[20] For reference, the impact sensitivity of TNT was measured as $h_{50 \%}>275 \mathrm{~cm}$. 